\title{
Incidentes críticos de violencia urbana vinculados al radicalismo islamista en España: simulación y análisis de un escenario*
}

\author{
Critical incidents of urban violence linked to Islamist \\ radicalism in Spain: simulation and analysis of a scenario
}

Recibido: 02 de octubre de 2013 | Aceptado: 25 de septiembre de 2015

\author{
Manuel Moyano PaCheCO ** \\ Universidad de Córdoba, España \\ Humberto Manuel Trujillo Mendoza*** \\ Universidad de Granada, España
}

doi:10.11144/Javeriana.upsy15-1.icvu

Para citar este artículo: Moyano, M., \& Trujillo, H. M. (2016). Critical incidents of urban violence linked to Islamist radicalism in Spain: simulation and analysis of a scenario. Universitas Psychologica, 15(1), 193-204. http://dx.doi.org/10.11144/Javeriana.upsy15-1.icvu

* Artículo de investigación científica y tecnológica. Este artículo, no tuvo financiación.

** Doctor en Psicología y profesor del área de Métodos de Investigación y Diagnóstico en la Facultad de Ciencias de la Educación

**** Doctor en Psicología y catedrático de Metodología de las Ciencias del Comportamiento en la Facultad de Psicología

\section{RESUMEN}

La amenaza del radicalismo islamista y del terrorismo derivado ideológicamente se han convertido en problemas estructurales que van a perdurar en los próximos años, sin visos de tener una solución a corto plazo. En el contexto de la Unión Europea, y en España en particular, existen indicadores que hacer pensar en la posibilidad de que en un futuro próximo se intensifiquen los incidentes críticos de violencia urbana anclados en un conflicto de identidades culturales interpretadas en clave Islam-Occidente. Este hecho, además de ser negativo para la convivencia y la cohesión social, podría ser aprovechado por las estrategias globales de los movimientos islamistas más extremos. Con el fin de llevar a cabo medidas proactivas que minimicen la polarización social y la radicalización de la ciudadanía, se simula un escenario a modo de hipótesis plausible y se evalúa desde una perspectiva psicosocial mediante un análisis DAFO (debilidades, amenazas, fortalezas y oportunidades). Se concluye respondiendo a algunas preguntas que pueden considerarse de utilidad para la toma de decisiones de los responsables políticos y con unas líneas estratégicas básicas desde un punto de vista de la gestión de la seguridad pública.

Palabras clave

radicalización islamista; yihadismo; violencia urbana; análisis DAFO; estrategia; escenario

\section{A B S T R A C T}

The threat of Islamist radicalism and terrorism derived ideologically have become structural problems that will endure in the coming years, no sign of having a short term solution. In the context of the European Union, and in Spain in particular, there are indicators that suggest the possibility that in the near future will intensify the critical incidents of urban violence, anchored in Islam-Western cultural identities key conflict are numerous. This fact, in addition to being negative for coexistence and social cohesion could be exploited by the global strategies of the most extreme Islamist movements. In order to carry out proactive measures that minimize social polarization and citizenship radicalization, we simulate a scenario as a plausible hypothesis which is evaluated from a psychosocial perspective using a SWOT analysis (strengths, weaknesses, opportunities and threats). We concluded with the response to some questions that we consider useful for decision-making of policymakers and with a basic strategic lines from a point of view of the management of public security.

Keywords

Islamist radicalization; jihadism; urban violence; SWOT analysis; strategy; scenario 


\section{Introducción}

¿Hasta qué punto puede llegar a ser la violencia urbana ${ }^{1}$ un problema grave de seguridad de los Estados que conforman la Unión Europea, en general, y de España, en particular? ¿Cómo actuar en caso de que dichos actos de violencia tengan un trasfondo ideológico legitimado, en su discurso y en su práctica, por grupos radicales islamistas y con una estrategia de desgaste para conseguir ciertos objetivos? ¿En qué sé diferenciaría este hecho de lo que conocemos hasta ahora? ¿Cómo debemos abordar eventuales crisis de este tipo desde un punto de vista de la seguridad pública? Y sobre todo, icómo no debemos abordarla?

Si tenemos en cuenta tanto la dinámica interna de los países de nuestro entorno en cuanto a la integración de los musulmanes se refiere (prejuicio y discriminación, incidentes de conflicto, polarización grupal y amenazas a la cohesión), como el contexto internacional (conflicto entre Israel y Palestina, insurgencia en Irak, Afganistán, Pakistán y norte de África, influencia de Irán y Arabia Saudí sobre las diásporas de musulmanes europeos, cambios políticos en los países del Magreb, amenaza del autonominado Estado Islámico), la amenaza del radicalismo islamista, y del terrorismo derivado ideológicamente de dicha radicalización se ha convertido en un problema estructural que va a perdurar en los próximos años, sin visos de tener una solución a corto plazo (Dalgaard-Nielsen, 2010; Moyano \& Trujillo, 2013). En este contexto global, varios son los aspectos que nos hacen pensar en la posibilidad de que en un futuro próximo se intensifiquen en España los actos de violencia urbana (de diverso tipo, de mayor o menor magnitud, perpetrados individualmente o en grupo) con base en una ideología islamista radical. Este hecho es plausible, sobre todo teniendo en cuenta algunos

1 En el presente trabajo definiremos la violencia urbana, anclada en cualquier tipo de ideología, sea la que sea, como un tipo de violencia política que se manifiesta típicamente en las calles, habitualmente por grupos de personas, con objetivos instrumentales y generalmente plasmado en actos concretos, tales como disturbios, protestas, altercados, destrozos del mobiliario urbano, revueltas, peleas o agresiones violentas. de los indicadores, muchos de ellos relacionados entre sí, que se exponen a continuación.

En primer lugar, los antecedentes en el pasado más reciente de España de conflictos de carácter etnicista. Recuérdense, a modo de ejemplo, los sucesos de Mancha Real o El Ejido (Río, 2005). Sucesos de este tipo, interpretados en clave Islam y Occidente, podrían tener una repercusión muy diferente de la que tuvieron los casos anteriores y pueden verse agravados por numerosos factores, tales como los discursos políticos que entienden y gestionan la inmigración como un problema y la asocian a tópicos como la violencia, la inseguridad ciudadana, el sistema penitenciario, la marginalidad o el control de las fronteras, así como por el aumento demográfico de los musulmanes y su influencia sociopolítica.

En segundo lugar, el contagio social que puede producirse debido a movimientos sociales de cierta magnitud y que, a priori, son lejanos geográfica o ideológicamente. Así, a modo de ejemplo, en los meses de octubre y noviembre de 2005 fuimos testigos de unos acontecimientos que vinieron a convulsionar diferentes capitales europeas. Nos referimos a los actos violentos callejeros en distintas y significativas ciudades de Francia, Bélgica y Holanda, que se propagaron con rapidez inusitada desde el epicentro parisino. Los disturbios violentos que se vivieron ese otoño supusieron una llamada de atención tanto para políticos como analistas, sobre un nuevo fenómeno típicamente urbano que, por lo imprevisible de su evolución y la larga duración en el tiempo, podía llegar a tener consecuencias muy graves. Aunque el origen de los disturbios no fue debido a un conflicto interreligioso anclado en el radicalismo islamista, incidentes puntuales a lo largo de los mismos y determinados actores implicados así lo hicieron parecer.

Hechos como los citados pueden favorecer una percepción en la opinión pública de que es legítimo canalizar las frustraciones personales (sin duda en ocasiones motivadas por la injusticia, las condiciones de vida difíciles o el descontento por las políticas llevadas a cabo) de una forma violenta e indiscriminada y que, además, se puede conseguir impacto social y alcanzar ciertos objetivos. Todo ello 
con el apoyo de diferentes sectores políticos y sociales, dotándolo de un aura revolucionaria atractiva para ciertos grupos que, en último término, puede favorecer que se emitan comportamientos miméticos de este tipo por grupos radicales islamistas, similares en sus acciones pero bien diferentes en sus objetivos.

En tercer lugar, al relacionarlo con lo anterior, los recientes atentados terroristas de Boston, Londres y París nos han mostrado que estamos, de hecho, ante eventos terroristas poco convencionales que los acercan, de algún modo a actos de violencia urbana de extrema gravedad.

En cuarto lugar, el previsible aumento de los inmigrantes de segunda y tercera generación con posibilidades reales de participar activamente en la sociedad aumentará, así como la probabilidad de que ciertas minorías de los mismos opten por una acción política no convencional. En una sociedad donde la actual minoría de musulmanes cobra protagonismo político y presencia social, episodios frecuentes de violencia urbana podrían no convertirse en algo descabellado, entendiendo el terrorismo como una estrategia asimétrica de acción política en la que las bases van a jugar un papel importante en dotar de apoyo y legitimidad a las causas más radicales, y en donde la escala de acciones violentas puede tener diferente grado (McCauley \& Moskalenko, 2008; Paul, 2010). Además, el carácter clandestino de los grupos terroristas necesitará de plataformas sociales que respalden, legitimen y apoyen, con mensajes o acciones de diferente orden, el objetivo último del yihadismo. Es ahí donde el apoyo de la sociedad civil va a tener su influencia y donde el Estado deberá poner los medios para controlarla.

En quinto lugar, la existencia en España de entornos sensibles, guetificados y deprivados a la vez, donde se vive tensión multicultural de diferente tipo (Moyano \& Trujillo, 2014; Trujillo \& Moyano, 2008). Tomando como referencia a otros Estados, como por ejemplo Francia, hay algunos aspectos que nos diferencian. Por una parte, hasta ahora nuestro número de inmigrantes de segunda y de tercera generación no tiene las dimensiones que han caracterizado a nuestros vecinos franceses. Por otra, nuestra inmigración está repartida en mayor medida. En otras palabras, hay menos barrios marginales y menos guetificados, siendo los barrios marginales de las principales capitales de nuestro Estado poco parecidas a las banlieus parisinas. ${ }^{2} \mathrm{No}$ obstante lo anterior, la existencia en España de diferentes entornos vulnerables a la radicalización y donde se vive tensión multicultural es un hecho.

En sexto lugar, debido al carácter modulador y a la influencia que puede tener en ciertos comportamientos sociales, la inestabilidad económica en la que estamos inmersos en estos momentos no va a hacer sino generar un caldo de cultivo favorable a ciertos factores psicosociales de riesgo de radicalización. De este modo, la privación relativa de necesidades básicas, la afectación psicológica o la percepción de conflicto multicultural, o la competitividad por conseguir ciertos recursos, por citar algunos factores de riesgo, pueden favorecer la desconexión moral de ciertas personas y grupos sociales, facilitar la emisión de comportamientos violentos y, por tanto, dificultar la regulación social por otras vías pacíficas (Moyano \& Trujillo, 2012; Sabucedo, Durán, \& Alzate, 2010; Trujillo, Jordán, Gutiérrez, \& González-Cabrera, 2009).

Entendemos que una juventud que tenga que demorar forzosamente su independencia, escasas posibilidades de incorporarse al mundo laboral, imposibilidad de asumir responsabilidades familiares

2 En 1995 se estrenó en Francia un film titulado La Haine (El odio) en el que se narraba, de forma ilustrativa, un día en la vida de tres jóvenes (un judío, un árabe y un negro) en un barrio marginal de París, Les Muguets: tres amigos deambulando por las calles, desentrañando en agudos diálogos sus dudas y sus miedos, en medio de la miseria, las drogas y la delincuencia. Esta fábula de los suburbios ejemplificaba las tensas revueltas vividas a comienzos de los noventa a raíz de los malos tratos recibidos por un joven musulmán a manos de la policía y cómo los jóvenes se debaten entre el odio a un sistema que no los incluye (racismo, discriminación, paro) y la falta de rumbo vital que los haga salir de esa situación. El film, dirigido por Mathieu Kassovitz, tiene un leit motiv que se repite en varias ocasiones. Una voz en off narra las siguientes palabras ejemplificadoras de cómo la sociedad parece mirar para otro lado ante un problema social que está ahí, latente: "Esta es la historia de una sociedad que se derrumba. Según se va derrumbando, se repite sin cesar, para tranquilizarse: hasta ahora todo va bien, hasta ahora todo va bien... Pero lo importante no es la caída, sino el aterrizaje". Casi veinte años después de aquella influyente película, el mensaje continúa vigente en nuestro país vecino (y por extensión en el nuestro), al que siempre hemos tenido como referente para ver lo que debemos y no debemos hacer en lo que a integración de inmigrantes se refiere. 
propias, así como una disposición de tiempo libre (generalmente asociada al desempleo) inversamente proporcional a unos objetivos personales propios y adaptativos a mediano o largo plazo, va a estar relacionada con la posibilidad de involucrarse en actividades delictivas en general $y$, en actividades de violencia urbana en particular.

En séptimo lugar, y muy relacionado con lo anterior, el papel que pueden desempeñar otros grupos sociales en futuros cambios sociocomportamentales de los grupos de radicales islamistas. Especialmente significativa es la influencia que pueden tener inmigrantes que hayan pertenecido en su historia previa a maras o pandillas. Es sabido que en la actualidad un gran número de jóvenes centroamericanos ha venido a España y, en numerosas ocasiones, su grado de afectación psicológica es elevado, estando un gran número de ellos, ya en su país de origen, bajo control de sectas religiosas o grupos manipulativos de distinta índole. En España no tienen líderes espirituales, siendo previsible, además, que aumente su estado de afectación psicológica. De este modo, tenemos que si en su país ya eran vulnerables a la captación por líderes mareros y religiosos, en España lo pueden ser también por otro tipo de líderes (Trujillo, Ramirez, \& Alonso, 2009). Dos conclusiones se pueden sacar de lo anterior. Por un lado, que los líderes yihadistas pueden sacar partido de este hecho en un futuro próximo. Por otro, que estos jóvenes centroamericanos pueden influir en las nuevas pautas de comportamiento grupal de los radicales islamistas, tales como la cohesión social, la jerarquía, los ritos o los conflictos asociados a la apropiación y el control de determinados espacios urbanos (territorialidad). En ese sentido, sería especialmente preocupante que determinadas zonas urbanas lleguen a ser ocupadas parcial o totalmente por los radicales.

En octavo lugar, y relacionado con lo anterior, el papel que desempeñan Ceuta y Melilla, enclaves simbólicos y estratégicos que nos vinculan inequívocamente con el mundo musulmán y punto de mira de la estrategia global del islamismo radical y a medio y largo plazo del terrorismo yihadista. Determinados incidentes críticos de violencia urbana que pudieran darse en dichas ciudades entre la población civil, asociados a la diversidad religiosa y cultural de las mismas, y de los que hay antecedentes, podrían ser instrumentalizados y magnificados en la dialéctica que utilizan los radicales para influir en las percepciones y, por ende, polarizar a los grupos sociales no ya a nivel local, sino a nivel global. Todo esto, de nuevo, con el fin de conseguir réditos estratégicos diversos. Los líderes radicales islamistas, entendiendo su estrategia como asimétrica, son bien conocedores de las oportunidades que un movimiento social de apoyo y adhesión en Ceuta y Melilla podría tener para dividir a la población civil (Llamas, 2011; Moyano \& Trujillo, 2013).

En noveno lugar y, teniendo en cuenta que el mejor predictor para que algo ocurra es saber si ha sucedido antes y en qué condiciones, es necesario decir que en los últimos meses se han vivido preocupantes actos de violencia asociada al hecho religioso en importantes ciudades europeas, así como una considerable tasa de repetición de actos de violencia urbana en los que se han visto implicados musulmanes europeos, bien como actores de la violencia, o lo que es igual de preocupante, como receptores de la misma (EUMC, 2006; Kruglanski, Crenshaw, Post, \& Victoroff, 2008; Trujillo \& Moyano, 2009). Así, se han vivido incidentes críticos en ciudades como Copenhague, Ámsterdam, Oslo, Marsella o Rótterdam, por citar algunas, y más recientemente en Malmö, Estambul, Lyon, Bruselas, Londres o Estocolmo. Incidentes que han sido asociados con el célebre asunto de las caricaturas de Mahoma pasando por disturbios urbanos de diferente tipo, como profanaciones de tumbas o ataques a mezquitas, morabitos y otros símbolos religiosos. En nuestro país, como decíamos antes, tanto en Ceuta y Melilla, como en otras ciudades, se han vivido también diferentes incidentes de ese tipo.

Por último, es previsible que la situación de violencia política en Oriente Medio y en el Magreb se intensifique en los próximos meses. Este hecho va a suponer un elemento crucial a tener en cuenta tanto en la política interior como exterior de los países de nuestro entorno. Las revueltas que surgieron en el mundo árabe desde finales de 2010, motivadas en principio por el deseo de reformas democráticas, justicia y avances sociales, se han visto truncadas 
en algunos casos. Si bien en cada país las movilizaciones han tenido una deriva y no se pueden realizar valoraciones generales, el retorno del Islam a la política, la potencial implantación de la sharia, así como la desvertebración de Estados otrora menos benevolentes con movimientos sociales como Hermanos Musulmanes, Tabligh o Hizb ut-Tahrir, nos hace suponer que ciertas áreas geográficas pueden tornarse en caldos de cultivo favorables al Islam radical. Las victorias de los islamistas en las elecciones celebradas tras las revueltas en Túnez, Marruecos y Egipto son una constatación de que tales movimientos sociales están organizados, vertebrados y que disponen de recursos logísticos. Y esta vez han sido las urnas las que han demostrado el apoyo y la simpatía que despiertan entre la base de la pirámide. Las guerras civiles (p. ej. en Siria) y el riesgo de que surjan estados fallidos en la región son escenarios poco reconfortantes más reales que ficticios en estos momentos (véase el caso del autodenominado Estado Islámico). Y es que, aunque el anhelo democrático es esperanzador para todos, los procesos de cambio político no van a ser ni rápidos, ni fáciles.

Partiendo del contexto anteriormente descrito, los objetivos del presente trabajo son los siguientes: (1) describir un escenario ficticio, a modo de hipótesis plausible, basado en incidentes críticos de violencia urbana anclados en un conflicto de identidades culturales interpretadas en clave IslamOccidente; (2) evaluar dicho escenario mediante un análisis DAFO (debilidades, amenazas, fortalezas y oportunidades); (3) responder a algunas preguntas útiles para la toma de decisiones de los responsables políticos, y (4) proponer unas líneas estratégicas básicas sobre lo que se debe y lo que no se debe hacer ante circunstancias de este tipo, desde un punto de vista de la gestión de la seguridad pública.

\section{Simulación de un escenario hipotético}

Desde un punto de vista preventivo, debemos prepararnos para lo peor, elaborando y poniendo a prueba lo que Lowenthal (1993) denominó hipótesis imposibles. Dicho autor pronosticaba la importancia de adaptarse a las nuevas amenazas de la seguridad, introduciendo el concepto de lucha contra lo increíble para enfatizar la importancia de anticiparse a las acciones que pudieran constituirse en amenazas reales para los Estados. De este modo, trabajar sobre hipótesis imposibles es simular situaciones imaginarias, inverosímiles o extremas, en las que la suerte de los acontecimientos pudiera ser tan inesperada que sorprendiera a las autoridades políticas decisoras, y por ende, a los ciudadanos. De forma genérica y tentativa, en este trabajo describiremos el siguiente escenario a modo de hipótesis imposible.

Consideramos que todos los indicadores expuestos hacen pensar que es previsible un futuro próximo donde nos encontremos con una sociedad debilitada por la crisis económica, con una bolsa de inmigrantes de segunda y tercera generación en aumento (de la cual un importante número son musulmanes, muchos de ellos deprivados), donde conviven grupos sociales con identidades sociales ciertamente heterogéneas (en ellos, el Islam radical puede desempeñar un papel clave para cohesionar a ciertos grupos) y donde existen numerosos contextos potencialmente conflictivos (zonas guetificadas, Ceuta y Melilla) en lo que a convivencia multicultural se refiere.

Esta situación inicial, unida a otros factores eventuales que pudieran concurrir (actos de discriminación real o percibida, atentados terroristas, violencia política en Oriente Medio), va a favorecer sin duda que, asociado o no a elicitadores de diversa índole, se puedan dar episodios más o menos continuos y más o menos graves de violencia urbana anclados en un conflicto de identidades religiosas y culturales diferentes, interpretados en clave Islam y Occidente.

Como reacción a este hecho, se dará, como casi siempre ocurre en situaciones de crisis, una respuesta más o menos espontánea por parte de la sociedad en general (grupos sociales, administración pública, medios de comunicación). Puede darse el caso de que, además, el Estado se vea - con sus medios, recursos y procedimientos - impotente para hacer frente a esta situación, con la consiguiente imagen de vulnerabilidad que esto pudiera transmitir a los actores implicados. 
Pero además, es tremendamente negativo por la influencia que podría tener, sería posible que, unido a la hipotética ineficacia del Estado para regular la situación de forma pronta mediante la mediación y los recursos policiales, fueran ciertos sectores de la sociedad civil los que entraran en una dinámica de violencia para vengar ciertos hechos o mostrar abiertamente comportamientos discriminatorios, con la casi inevitable escalada del conflicto civil, la polarización grupal y la consiguiente radicalización de unos y otros.

En resumidas cuentas, a raíz de esta serie de acontecimientos, y modulado por las respuestas policiales, los medios de comunicación social y los mensajes institucionales, es fácil que se reproduzca una escalada de acción-reacción, tensión grupal y cierto caos social. Entendiendo estos procesos de polarización grupal como movimientos sociales en los que no todos los que apoyan una ideología radical serán capaces de cometer acciones violentas, pero sí de actuar de diferente manera con un objetivo instrumental, estos acontecimientos podrían ser tremendamente rentabilizados en la estrategia global del terrorismo yihadista para favorecer la victimización, la justificación de la violencia y la percepción de un conflicto inevitable entre Islam y Occidente. Los líderes radicales podrían también pretender el mantenimiento de acciones extremas de protestas y disturbios con el fin de forzar la represión estatal. En caso de que ésta se produjera, podríamos llegar a una situación en la que un gran número de los musulmanes que viven en el Estado se pusiera del lado de los radicales islamistas, en otras palabras, podrían salir reforzados ante una crisis de este tipo. Así, aún en el caso de que dicha crisis fuera resuelta de una manera más o menos rápida y eficaz, podría ser instrumentalizada estratégicamente por los líderes islamistas de una forma particularmente grave. Todo ello para conseguir objetivos a largo plazo en la estrategia del yihadismo global y que se pueden plasmar en la polarización de los grupos y la división de la sociedad.

Es difícil predecir todos los actores y los procesos que podrían darse en un escenario como el propuesto. Sin embargo, entendemos que todas las acciones dirigidas a una prevención del conflicto y a la anticipación del mismo antes de que suceda serán positivas. No olvidemos que una vez que el problema aparece, el tiempo será un factor que juegue en nuestra contra. Hecha esta puntualización, pasaremos a continuación a realizar un análisis DAFO del escenario hipotético.

\section{Evaluación del escenario a través de la matriz DAFO: debilidades, amenazas, fortalezas y oportunidades}

Básicamente DAFO proviene de la palabra SWOT, acrónimo en inglés de Strenghts, Weakness, Oportunnities and Threats. Podemos definir el análisis DAFO como un análisis del balance de fuerzas de un sistema ante una situación concreta (en este caso, ante el escenario hipotético anteriormente expuesto). El objetivo fundamental es concretar en una matriz resumen o un gráfico las debilidades y amenazas que hacen vulnerable un sistema, así como sus fortalezas y oportunidades.

El procedimiento para realizar una matriz DAFO puede ser de diferentes formas. En ocasiones se realizan grupos de discusión, brainstormings o Delphis con expertos.

Además, puede ser complementado con otras técnicas como el método de impactos cruzados, donde la estadística y la probabilidad se convierten en grandes aliadas del análisis que posibilitan el manejo de datos cuantitativos y la ulterior extracción de conclusiones (Smida, 2010). Este tipo de análisis, práctico y viable, ofrece una información valiosa, especialmente en las fases iniciales del análisis estratégico y en la toma de decisiones (Hill \& Westbrook, 1997; Pickton \& Wright, 1998).

Debemos tener en cuenta, que un análisis DAFO no es ni más ni menos que un modelo estático de la realidad en un momento temporal puntual. Por tanto, no es algo que perdure en el tiempo, sino modificable según las circunstancias en las que concurra. Dicho esto, pasaremos a definir cada uno de los componentes y a proponer los factores que consideramos que deben tenerse en cuenta en cada uno de los mismos. 


\section{Debilidades}

Podemos definir las debilidades como las limitaciones que constituyen obstáculos para la consecución de los objetivos. Al constituir una dificultad para responder a las oportunidades y amenazas, éstas deben ser controladas y superadas para conseguir minimizar su impacto. Se trataría de responder a las siguientes preguntas: icuáles son nuestros puntos débiles? y ¿dónde podemos mejorar? Entre otras, podemos citar las siguientes debilidades: (1) previsible aumento de la polarización intergrupal; (2) existencia de entornos sensibles en nuestro Estado, guetificados y deprivados a la vez, donde se vive tensión multicultural; (3) conflicto social novedoso; (4) reacción, a veces algo desproporcionada, por parte de los cuerpos y fuerzas de seguridad; (5) falta de cohesión y unidad en los principales partidos políticos ante una crisis de esta magnitud; (6) carencia de protocolos de coordinación entre los organismos dependientes de la administración; (7) respuestas institucionales tardías; (8) discursos legitimadores de la violencia por parte de ciertos sectores minoritarios antiintegración en mezquitas, en el seno de las comunidades islámicas y otros entornos; (9) heterogeneidad ideológica y política del Estado; (10) islamismo radical como ideología que va favorecer la cohesión y la lealtad de los grupos que lo apoyan; (11) existencia de prejuicio y discriminación hacia los musulmanes; (12) existencia de aspectos controvertidos y no resueltos sobre la integración del Islam en Occidente: puntos de fricción; (13) carencia de una estrategia clara y consistente en la prevención del radicalismo islamista.

\section{Amenazas}

Las amenazas son aquellas situaciones o circunstancias del entorno potencialmente desfavorables y limitantes, y por tanto, no deseables, entre otras cosas, porque no se está preparado para afrontarlas. El sistema debe enfrentarse a las mismas con el fin de minimizar los daños y adaptarse, ya que pueden impedir el desarrollo de una estrategia concreta, o bien, reducir su efectividad. Estas amenazas son: (1) disturbios de carácter etnicista que puedan ser interpretados en clave Islam-Occidente; (2) contagio social que puede producirse debido a movimientos sociales de cierta magnitud y que, a priori, son lejanos geográfica e ideológicamente; (3) antecedentes de actos terroristas poco convencionales que los acercan, de algún modo, a actos de violencia urbana de extrema gravedad (p. ej. Bombay, Toulouse y Montauban, Boston, Londres); (4) previsible aumento de inmigrantes de segunda y tercera generación; (5) papel que pueden desempeñar ciertos grupos sociales en futuros cambios sociocomportamentales de los grupos de radicales islamistas (p. ej. movimientos antisistema, mareros); (6) antecedentes preocupantes en ciudades europeas de actos violentos asociados al hecho religioso; (7) actuaciones irresponsables de los medios de comunicación social; (8) tensiones en el contexto internacional, especialmente Oriente Medio (Iraq, Afganistán, Palestina, Irán); (9) efectos de la crisis económica: aumento de las tasas de desempleo, privación relativa, necesidades vitales no cubiertas, percepción de injusticia social; (10) el papel geoestratégico que desempeñan Ceuta y Melilla; (11) atrincheramiento, toma de infraestructuras e instituciones, incluso ciertas zonas urbanas con evidente valor emocional e identitario para los grupos sociales implicados; (12) efectos globalizadores de Internet y las nuevas tecnologías; (13) grupos o movimientos sociales, en principio ajenos al radicalismo islamista, que apoyen o legitimen posibles disturbios asociados al mismo, bajo una estrategia de populismo radical; (14) aumento de la población musulmana en Ceuta y Melilla, así como en determinados guetos; (15) auge de partidos de extrema derecha.

\section{Fortalezas}

Las fortalezas son los elementos favorables o puntos fuertes internos al sistema. Se trata de la posición favorable que el sistema tiene en diferentes aspectos que le permiten responder eficazmente ante determinadas oportunidades y amenazas. Se trataría de responder las siguientes preguntas: ¿en qué somos más fuertes? ¿En qué nos podemos apoyar? Podemos enumerar las siguientes fortalezas: (1) asimetría de fuerzas: mayor cantidad de recursos y capacidad del Estado ante potenciales conflictos; (2) en estos momentos, todavía no puede hablarse en sentido 
estricto de una segunda y tercera generación de inmigrantes en España, aunque es cuestión de tiempo; (3) no hay guetos de tanta envergadura como, por ejemplo, en Francia. Además, la población inmigrante está más repartida.; (4) elevado nivel de experiencia y capacitación de las fuerzas y cuerpos de seguridad del Estado; (5) existencia de dispositivos con lecciones aprendidas sobre lo que ha ocurrido en otras ocasiones ante fenómenos semejantes, como por ejemplo con el terrorismo callejero de ETA; (6) iniciativas a nivel europeo para prevenir el prejuicio y la discriminación hacia los musulmanes, así como la radicalización islamista.

\section{Oportunidades}

Las oportunidades son situaciones o circunstancias del entorno potencialmente favorables, es decir, todo aquello que puede suponer una ventaja o una posibilidad para mejorar la eficacia en la gestión de los recursos y alcanzar los objetivos del sistema. Podemos citar las siguientes: (1) disponibilidad de medios para concienciar y asesorar a los responsables políticos; (2) llevar cuanto antes ante la justicia a los responsables de hechos violentos; (3) oportunidad de aislar a los violentos si las comunidades musulmanas en general y ciertos grupos en particular (p. ej. Tabligh, Justicia y Espiritualidad) rechazan explícita y contundentemente el uso de la violencia y, por tanto, el yihadismo; (4) disponibilidad de medios para afianzar ante la opinión pública y la ciudadanía los valores democráticos, la condena de la violencia y el respeto a la diversidad; (5) posibilidad para hacer visibles y admirados socialmente a los líderes locales y agentes sociales de las comunidades musulmanas que estén claramente a favor de la integración; (6) disponibilidad de medios para aprovechar el asociacionismo y las relaciones institucionales con el fin de transmitir mensajes de unidad; (7) capacidad de realizar labores de inteligencia.

En la Figura 1 se representa, en forma de mapa perceptual, el análisis DAFO.

Varios son los aspectos a destacar en un examen preliminar de la matriz DAFO.

En primer lugar, todos los factores que se enumeran podrían clasificarse según los agentes im- plicados y agruparse en otros factores más incluyentes. Así, la mayoría de los factores se derivan de la influencia y la responsabilidad de la sociedad civil en general, de las administraciones del Estado (cuerpos y fuerzas de seguridad, servicios sociales, sistema educativo), medios de comunicación social, aspectos geográficos y de zonificación del entorno urbano y comunidades musulmanas.

En segundo lugar, al haber simulado un escenario de crisis, encontramos que hay un cierto desajuste debido a que las limitaciones del sistema (debilidades y amenazas) son más numerosas que las potencialidades (fortalezas y oportunidades). Como puede apreciarse, ante una crisis de este tipo hay bastantes más aspectos negativos que positivos que deben ser compensados.

En tercer lugar, esta matriz DAFO podría completarse con otras etapas analíticas de naturaleza cuantitativa asignando pesos numéricos a los factores, comparando las fuerzas positivas con las negativas, así como el contexto psicosocial y político interno con el externo. También podrían ordenarse utilizando diferentes criterios como, por ejemplo, la prioridad con la que deberían atenderse las distintas dimensiones o entradas del sistema. De cualquier forma, la participación de expertos (procedentes de diferentes ámbitos profesionales o académicos) sería recomendable para llevar a cabo este proceso.

\section{Respuestas a algunas preguntas de interés para la toma de decisiones}

En el análisis de este escenario nos proponemos derivar algunas implicaciones políticas, policiales y de seguridad pública partiendo de la estructura matricial y del mapa anteriormente expuesto. Para ello, trataremos de responder brevemente las siguientes preguntas, que consideramos de interés para decisores y responsables políticos.

¿Hasta qué punto pueden ser los actos de violencia urbana, en general, un problema grave de seguridad para los Estados?

Podemos afirmar que pueden serlo. En los últimos años y en diferentes partes del mundo, incluido el 


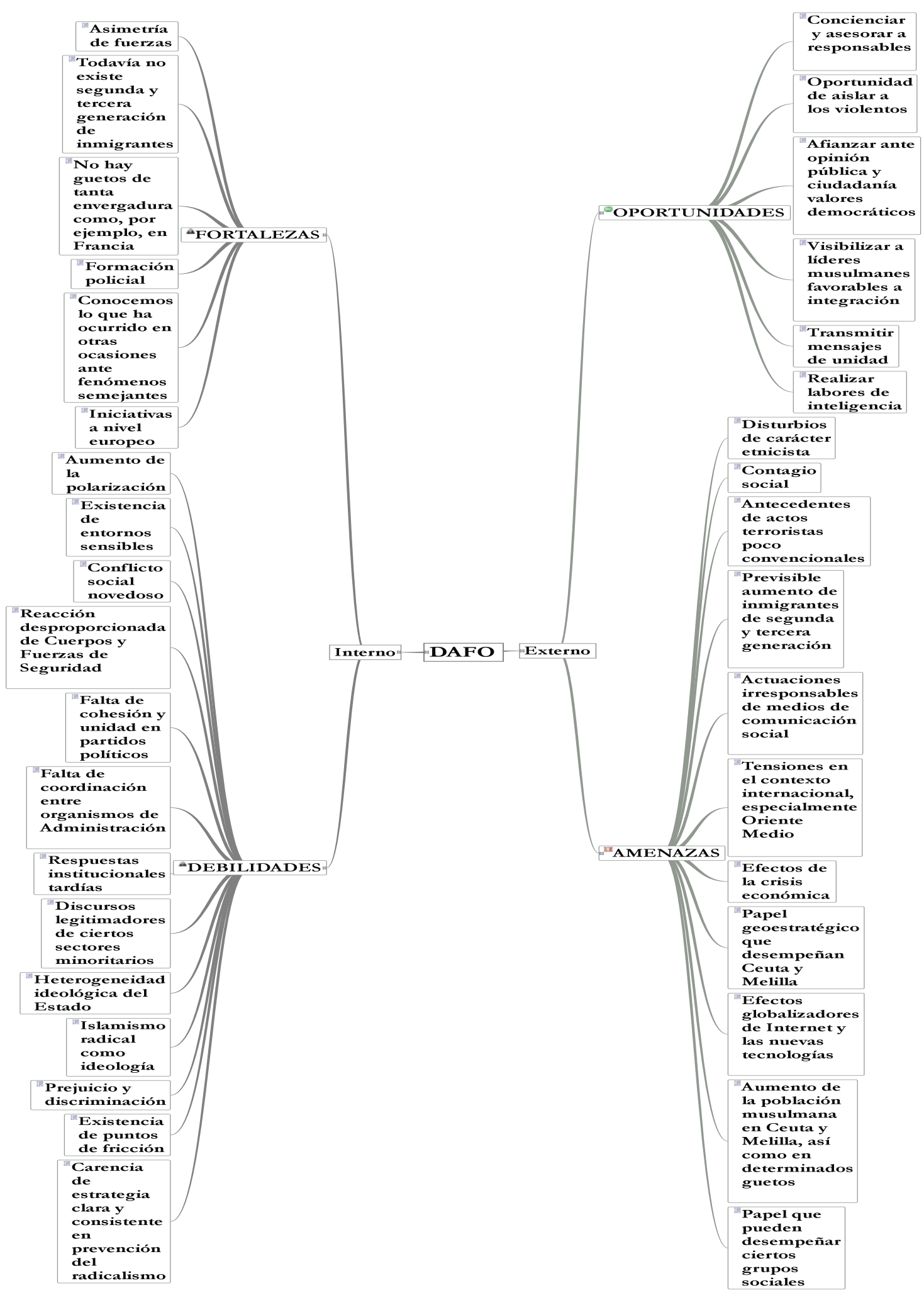

Figura 1. Mapa del análisis DAFO

Fuente: elaboración propia 
Estado español, hemos asistido a diferentes actos de violencia urbana de mayor o menor intensidad. Así, hemos presenciado disturbios; graves actos terroristas, semejantes en su ejecución a las acciones típicas de guerrilla urbana; pasando por revueltas violentas de carácter étnico, o violencia interpersonal. En un mundo cada vez más globalizado y con unos medios de comunicación trasnacionales, una crisis puntual puede convertirse en global en cuestión de minutos, por lo que no hay que subestimar amenazas aparentemente inocuas.

¿Serían diferentes los actos de violencia urbana anclados en el radicalismo islamista de otros tipos de violencia urbana asociados a otras ideologías?

En general, una de las características de las revueltas antisistema y de los disturbios de carácter etnicista es que suelen ser sofocadas en relativamente poco tiempo. En pocas ocasiones han durado más de unos días. Tras las intervenciones policiales y las mediaciones oportunas, el asunto se cierra, deja de aparecer en los medios y el contrato social se sella de nuevo dejando latente movilizaciones que periódicamente convulsionan importantes ciudades con una trascendencia mediática global. Sin embargo, consideramos que hipotéticas crisis de violencia urbana ancladas en el radicalismo religioso en general, y en el radicalismo islamista en particular, podrían ser meridianamente diferentes que cualquier otro tipo de acto violento típicamente urbano que pudiera estar asociado a otro tipo de ideología. Por tanto, el manejo de dichas situaciones también debe de tener sus especificidades.

\section{¿Cuáles podrían ser los detonantes}

de actos violentos de ese tipo?

Como ya hemos comentado, el principal detonante de disturbios de este tipo pueden ser hechos que se consideren agravios al mundo musulmán o al Islam (ataques a mezquitas, caricaturas de Mahoma). También se han repetido, en numerosas ocasiones, asociadas a reivindicaciones concretas, más o menos razonables, en principio pacíficas, que posteriormente derivaron en disturbios urbanos.
De especial trascendencia es el conflicto de Israel y Palestina, la violencia política en Siria, Afganistán o Iraq, o la situación geoestratégica tras la mal denominada, de forma precipitada, Primavera Árabe. Diferentes acciones pueden incrementar los sentimientos de victimización por parte de los musulmanes occidentales y poner las cosas realmente fáciles a aquellos que pretenden manipular percepciones al considerar un conflicto sin duda grave, pero puntual y localizado geográficamente en un contexto concreto, como algo global y con el que "todos los musulmanes" deben verse lealmente comprometidos. Es particularmente grave este tipo de acontecimientos, ya que eventuales actos violentos por parte de radicales islamistas podrían incluso legitimarse desde sectores sociales que en principio nada tienen que ver con las comunidades musulmanas.

¿Qué medidas preventivas deben seguir desarrollándose para prevenir el radicalismo islamista $y$, por tanto, incidentes de violencia urbana asociados al mismo?

En los últimos años se han ido adoptando en las sociedades occidentales una serie de medidas a nivel policial y de inteligencia, pero, además, se han desarrollado medidas para potenciar la integración de los musulmanes en la sociedad en torno al empleo, la educación, la participación social de las comunidades musulmanas y el diálogo interreligioso. Es necesario incidir en las iniciativas para combatir la islamofobia y favorecer la cohesión. No debemos olvidar que el objetivo último de esta estrategia no es otro que la integración y la formación de los grupos sociales y las personas en riesgo de radicalización. Cada vez más, esas medidas están coordinadas a nivel de Unión Europea e instituciones internacionales, intercambiando buenas prácticas y favoreciendo un abordaje común.

\section{Conclusiones}

El uso que se le suele dar al análisis DAFO es variado y versátil. Proveniente del ámbito de la economía y la administración de empresas, en ocasiones sólo 
se utiliza para tener una visión general del sistema o la situación objeto de estudio, y en otras para plantear una estrategia que podrá ser ofensiva, defensiva, de contingencia o de supervivencia. En definitiva, es una herramienta útil que la psicología aplicada a la intervención social puede utilizar en escenarios hipotéticos, como el expuesto, donde haya que tomar decisiones importantes en un corto periodo de tiempo. Somos conscientes de la cautela que debemos mostrar al proponer estrategias antiterroristas concretas, especialmente ante hechos críticos que no han ocurrido y, que de hecho, no tienen por qué ocurrir. Mucho más teniendo en cuenta que cada vez se oyen voces críticas sobre las medidas antiterroristas que se vienen tomando por los responsables políticos y la necesidad de que realmente sean efectivas, no perjudiciales a largo plazo y basadas en la evidencia científica. En otras palabras, que de alguna manera se controle la incertidumbre que puede generar el actuar mediante programas concretos con consecuencias imprevisibles y desconocidas para la seguridad pública (Ginges, Atran, Sachdeva, \& Douglas, 2011; Leblanc \& Robert, 2012; Lum, Kennedy, \& Sherley, 2008). Sin embargo, consideramos que al menos es posible establecer unos límites estratégicos ante escenarios similares al descrito. Como podremos ver, muchas de las medidas que proponemos se nutren directamente de la matriz DAFO realizada.

Los siguientes aspectos deben considerarse prioritarios ante una crisis hipotética:

- Es necesario asesorar y concienciar a los responsables políticos de la trascendencia que una crisis de este tipo podría tener y la importancia de estar preparados para la misma.

- Evitar, en la medida de lo posible, una acción desproporcionada por parte de los cuerpos y fuerzas de seguridad, con la consiguiente escalada de acción-reacción y los discursos victimistas posteriores.

- Es importante no gestionar el asunto como un choque cultural o religioso. En ningún caso se debe menospreciar, agraviar o amenazar a la religión y condenar claramente los mensajes que vayan en esa dirección. Provocaciones gratuitas sólo conseguirían cohesionar más a los violentos, legitimar la violencia y la victimización. Los posibles símbolos y valores morales que cohesionan al Estado son mucho más heterogéneos y menos unitarios que el Islam, que interpretan de una forma distorsionada los violentos. Lo único que podemos conseguir mediante humillaciones (reales o percibidas) gratuitas es ampliar la categoría de identidad social de los violentos y hacerla más incluyente, en lugar de hacerla más excluyente para aquellos que en teoría no legitiman la violencia en nombre del Islam.

- Tratar de evitar convertir cualquier acontecimiento de este tipo en algo global, con el que se identifiquen musulmanes de otros entornos, percibiéndolo como una "lucha contra ellos".

- Es necesario que los medios de comunicación social, y más concretamente sus responsables, sean colaboradores para participar de la comunicación estratégica que se debe emplear para minimizar este tipo de crisis.

- Es necesario visibilizar a los líderes, eventuales o no, que tengan actitudes favorables a la integración y discursos no violentes.

- Es necesario visibilizar el desarrollar buenas prácticas de cooperación entre las autoridades y la sociedad civil.

- Es necesario aislar a los violentos haciendo visibles las críticas de las comunidades musulmanas, por ejemplo a través de los líderes religiosos.

- Como medida disuasoria, detener y poner ante la justicia a los implicados cuanto antes.

- Proteger infraestructuras y entornos críticos que pudieran ser atacados, especialmente aquellos con valor emocional e identitario para los grupos implicados.

- Dentro de lo posible, aprovechar hipotéticos escenarios de este tipo para hacer labores de inteligencia.

- Control de los líderes eventuales radicales que puedan surgir.

- Consideramos que es de vital importancia prevenir la violencia social antes de que llegue 
a las calles. Si esto ocurre la percepción del conflicto multicultural se hará patente y las medidas preventivas que promuevan la paz y el respeto entre comunidades podrían dar paso a otro tipo de discursos que, según el contexto, podrían a su vez generar más radicalismo, plasmado en el auge de partidos de extrema derecha, legitimación de la violencia por ambas partes, desconfianza en aumento y un incremento en la popularidad de medidas reactivas de corte policial y discriminatorio.

\section{Referencias}

Dalgaard-Nielsen, A. (2010). Violent Radicalization in Europe: What We Know and What We Do Not Know. Studies in Conflict and Terrorism, 33, 797-814.

EUMC. (European Monitoring Centre on Racism and Xenophobia) (2006). Muslims in the European Union. Discrimination and Islamophobia. EUMC. Recuperado el 10 de enero de 2011 de http://fra. europa.eu/fra/material/pub/muslim/Manifestations_EN.pdf

Hill, T., \& Westbrook, R. (1997). SWOT analysis: It's time for a product recall. Long Range Planning, 30, 46-52.

Ginges, J., Atran, S. Sachdeva, S., \& Douglas, M. (2011). Psychology out of the laboratory: The challenge of violent extremism. American Psychologist, 66, 507-519.

Leblanc, L., \& Robert, M. (2012). Innovación psicosocial: aplicación de un plan de prevención de la delincuencia juvenil. Universitas Psychologica, 11 (4), 1125-1134.

Lowenthal, M. (1993). Intelligence Epistemology: Dealing with the unbelievable. International Journal of Intelligence and Counterintelligence, 6, 319-325.

Lum, C., Kennedy, L., \& Sherley, A. (2008). Is counterterrorism policy evidence-based? What works, what harms, and what is unknown. Psicothema, 20, 35-42.

Llamas, M. (2011). Melilla y el islamismo: luces y sombras. Madrid: Atanor Ediciones.

Kruglanski, A. W., Crenshaw, M., Post, J. M., \& Victoroff, J. (2008). What Should This Fight Be Called?
Metaphors of Counterterrorism and Their Implications. Psychological Science in the Public Interest, 8, 97-133.

McCauley, C., \& Moskalenko, S. (2008). Mechanism of political radicalization: pathways toward terrorism. Terrorism and Political Violence, 20, 415-433.

Moyano, M., \& Trujillo, H.M. (2012). Evaluación del riesgo de radicalización islamista en cuatro contextos de interés estratégico para España. Comunicación presentada en el III Congreso Internacional de Inteligencia, Barcelona, 12 de noviembre de 2012.

Moyano, M., \& Trujillo, H.M. (2013). Radicalización islamista y terrorismo. Claves psicosociales. Granada: Editorial Universidad de Granada

Moyano, M,. \& Trujillo, H.M. (2014). Intention of activism and radicalism among Muslim and Christian youth in a marginal neighbourhood in a Spanish city. International Journal of Social Psychology, 29, 90-120.

Paul, C. (2010). As a Fish Swims in the Sea: Relationships Between Factors Contributing to Support for Terrorist or Insurgent Groups. Studies in Conflict and Terrorism, 33, 488-510.

Pickton, D. W., \& Wright, S. (1998). What's swot in strategic analysis? Strategic Change, 7, 101-109.

Río, M. A. (2005). Dilemas políticos y de orden público: alcaldías y fuerzas de seguridad en disturbios etcnicistas. Política y sociedad, 42, 63-85.

Sabucedo, J. M., Durán, M. M., \& Alzate, M. (2010). Identidad colectiva movilizada. Revista de Psicología Social, 25, 189-202.

Smida, A. (2010). Hacia una memorización de los signos de los impactos cruzados entre variables estratégicas. Innovar, 20, 205-218.

Trujillo, H.M., \& Moyano, M. (2008). El sistema educativo español en la prevención de la radicalización yihadista. Athena Intelligence Journal, 3, 75-83.

Trujillo, H.M., Ramírez, J.J., \& Alonso, F. (2009). Indicios de persuasión coercitiva en el adoctrinamiento de terroristas yihadistas: hacia la radicalización violenta. Universitas Psychologica, 8, 721-736.

Trujillo, H.M., Jordán, J., Gutiérrez, J.A., \& GonzálezCabrera, J. (2009). Radicalization in Prisons? Field Research in 25 Spanish Prisons. Terrorism and Political Violence, 21, 558-579. 